\title{
ANALYSIS OF EXPRESSING AUDIENCES IN A CYBER-THEATER
}

\author{
Kang DongWan, Kay Huang and Jun Ohya
Graduate School of Global Information and Telecommunication Studies, Waseda University, Tokyo, 169-0051, JAPAN

\begin{abstract}
This paper studies how audiences should be expressed in a Cyber- theater, in which remotely located persons can direct plays as directors, perform as performers and/or see the performances as audiences through a networked virtual environment. It is noted that the audience effect has been widely acknowledged in the real-world theater: that is, the audience reaction has a significant effect on the acting of player and performance of the play itself. However, only a few works relevant to audiences in the Cyber Theater can be seen. This paper studies whether the audience effect exists also in the Cyber-theater. By constructing a system in which two actors are displayed a remotely located audience's avatar in which the audience can display his/her emotional actions, we clarified that interactions between the actors and audience are effective.
\end{abstract}

\section{INTRODUCTION}

The authors aim at achieving a virtual space that could let the users join as an actor, a director, or an audience from their homes or offices through networks. We call this system Cyber Theater [1]. Nowadays, it is very common to use 3dimensional motion capture to catch humans' actions. Therefore, humans' motion analysis and synthesis are widely researched. The authors developed a color image processing based method for estimating a person's postures [3],[4]. Most of these technologies, however, are applied to the actors, but there are not many research works related to the audience in a virtual space.

Slater et al. worked on a method that helps cure social phobia by using a virtual tool [2]. The related part of their research is their experiment. They arranged 8 avatars in a seminar room, and the speakers were made to make a short speech alone. However, their experiment is aimed at testing whether the speaker can feel the avatars exist as audiences, but not focus on the interactions between audiences and actors.

In this paper, we explore whether the audience effect exist also in the Cyber-theater. We construct a system in which two actors and one audience can join, where the audience's avatar is displayed to the screen the two actors can see. Experimental results demonstrate that interactions between the actors and audience are effective.

\section{REAL WORLD THEATER INTERACTION ANALYSIS}

In a real-world theater, there are certainly kinds of interactions between audience and actors, and of course, also between audience and audience. These interactions are deeply related to the success of concerts and/or plays.

Therefore, we have to clarify these interactions in real world to make the cyber-theater more similar to or more effective than real world.

\subsection{INTERACTION BETWEEN ACTORS AND AUDIENCE}

To obtain a specific idea about the interactions between audience and actors in real world, we visited some real actors who have experiences on the stage and conducted questionnaires.

From the result we found that the final purpose for actors to perform hard is in order to gain excellent responses from the audience. Besides, when the audiences give positive reactions, the actors feel pleased and want to perform better; but when the audiences show negative reactions, the actors feel upset and lose their confidence. In summery, we are sure that the relationship between actors and audiences is very important to achieve successful performances.

\subsection{INTERACTION BETWEEN AUDIENCE AND AUDIENCE}

In order to gain a specific idea about the interactions among audiences in real world, we ask some people who have been to a concert or show to fill out another questionnaire.

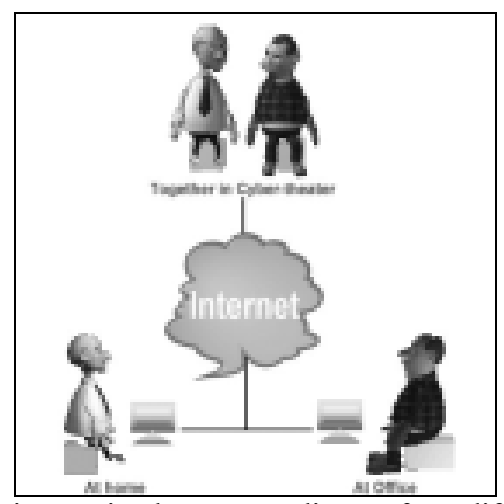

Figure 1. The interaction between audiences from different sites

According to the data we got from the questionnaire, it 
turns out that audiences also concern about the other audiences' actions even when they are watching the performance. Besides, they often do some kinds of actions just because other people do that Moreover, most audiences enjoy watching performances with the other people, because it is more fun to laugh together, cheer together or discuss with each other.

In addition, since there is only one person in one site in the cyber-theater, it is necessary to find out how to make this kind of interactions between audiences in different sites (Figure 1). For this reason, how to combine all of the audience into one space is also a very important theme.

\section{AUDIENCE OF CYBER-THEATER SCENARIO}

About cyber-theater, since it is a "theater", obviously it consists of actors and audiences. Note that cinemas can be included in cyber-theater, but in case of cinemas there is no interaction between actors and audience. This paper is concerned with theater arts such as concerts and plays.

Figure2 illustrates a conceptual system set-up for the audience study. In the screen, each audience can see avatars for all the audiences and avatars for the actors, where the audiences and actors are actually remotely located.

During the time each audience sees the actors' performance presented by the avatars in the screen, each audience can display his/her reactions through some interface to his/her avatar, where this paper uses a "push button" interface for displaying the audiences' reactions.

The actors can see the audiences' reactions by seeing the audiences' avatars displayed in the screen. Note that, about the actors' avatars, motion capture and avatar simulation are needed, but these technologies are still on-going projects; therefore, this paper does not detail these.

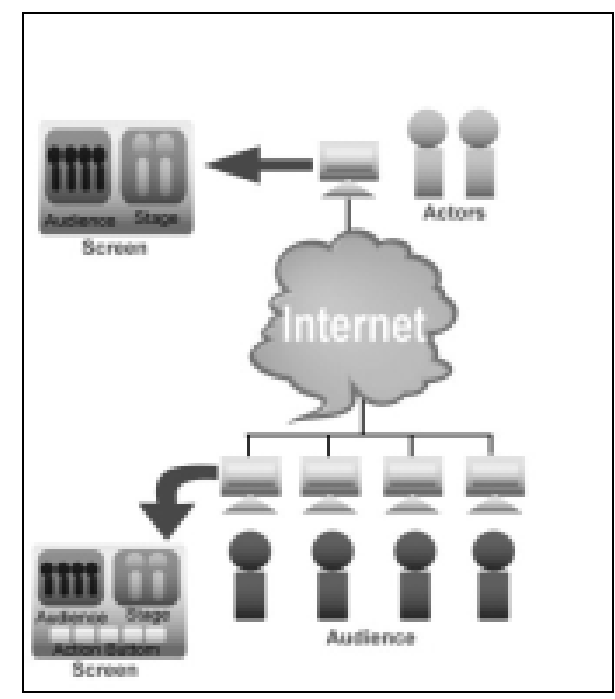

Figure 2. Illustration of the audience of cyber-theater scenario

The audiences' actions (reactions) to be displayed by the avatars should be based on real human audiences' actions in theater. To observe the actions of audiences in real world, we participated in some concerts, musical plays and movies.
However, since it is hard to record the actions of audiences in real world theaters, we invited some people to gather and watch a video together; and we video-taped the experience. Note that we had told them that the video-tapes were to be used only for research purposes.

\section{CONSTRUCTING AND ANIMATING AUDIENCE'S AVATARS}

In order to let the user who participates in cyber-theater feel that they are interacting with real people, it is very important that the actions of avatars are similar to real human's actions. In particular, the facial expressions of avatar and the motions of the upper part of the body are very important, because the audiences are sitting on the chairs in the theater.

Figure 3 is the main scene of the model system we constructed. In this model system, there are 10 people sitting on the chair and they can display 10 kinds of actions, including clap, laugh, talk, sleep, leave, bore, agree, disagree, surprise, clap, and laugh.

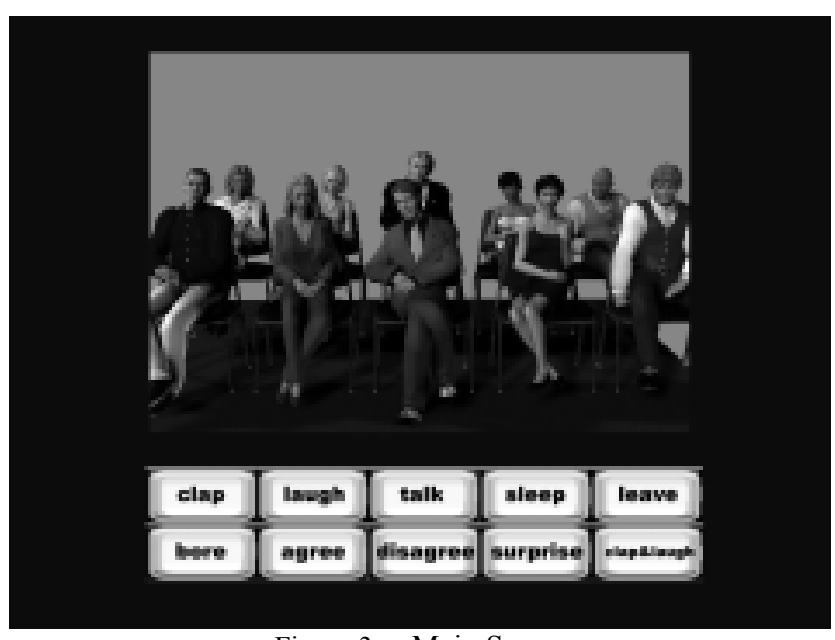

Figure 3. Main Scene

\section{EXPERIMENTAL RESULTS AND DISCUSSION}

The purpose for this experiment is to clarify whether the audiences' avatars can let the actors feel that they are presenting their performances to real people and whether the audiences' avatars can give influences on the actors' performances. In addition, we also want to obtain the audience's remarks on our model system.

\subsection{EXPERIMENTAL CONDITIONS}

The configuration of this experiment is shown in Fig. 4. We used a camera to videotape the performance of actors, and the videotaped performance was displayed on the audience's screen. In the audience's screen he/she can see the auditorium, stage (performance) and ten action buttons.

The audience can use the buttons to display his/her emotions. In addition, we used a projector to project the 
auditorium on to the wall so that the actors can look at the avatars while they are performing. In order to prevent actors and audience from interfering with each other; we used a board to separate them.

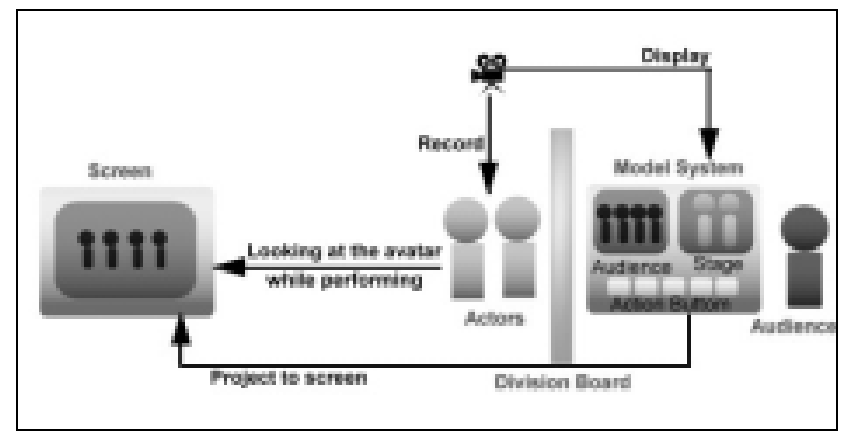

Figure 4. Illustration of the experimental Configuration

\subsection{EXPERIMENTAL PROCEDURE}

Groups each of which includes two or three persons are formed, where one or two persons are the performers (Fig.5), and the other one is the audience. As the performers, we invited 2 people from an entertainer company, and 8 people from a rakugo club of a university. Also we invited some amateur people who experienced to perform on the stage.

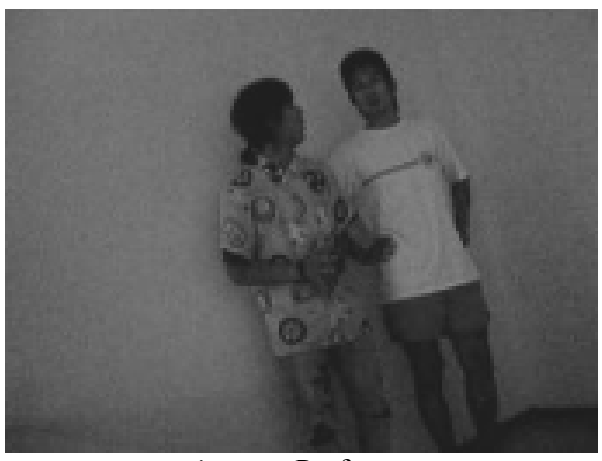

Figure 5. Performer

As described in Section 3, actors' avatars are not displayed in the screen due to technical difficulties. Instead, a video camera observes the actors, and the video sequence observed by the computer is displayed in the screen in real-time. Our specific experimental environment is shown in Fig. 6. Since the actors and audience share one room, a board separates the actors and audience so that they do not directly interact with each others audio-visually.

Performers are asked to perform for 5 10 minutes. When they are performing, they face to the avatars of audiences that are projected on the wall (Figs. 7 and 8). At the same time, the person who is the audience uses the model system and selects the action buttons based on how he/she feels about the on-going performance (Fig.9).

Before the experiment, we let the subjects fill out a questionnaire. Performers and audiences have different questionnaires. After the experiment, we let subjects fill out another questionnaire.

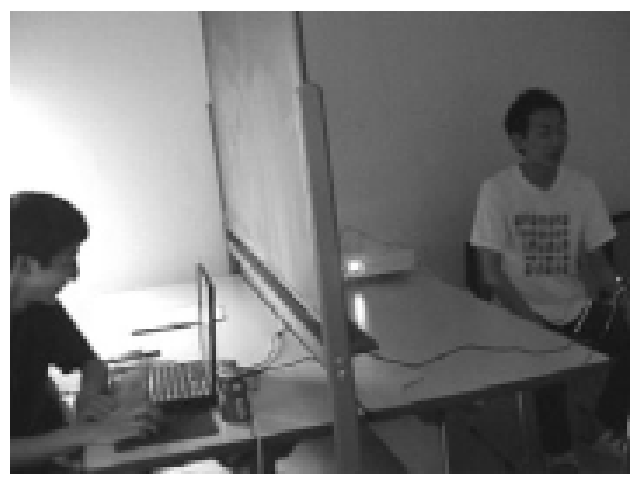

Figure 6. Actors and audiences

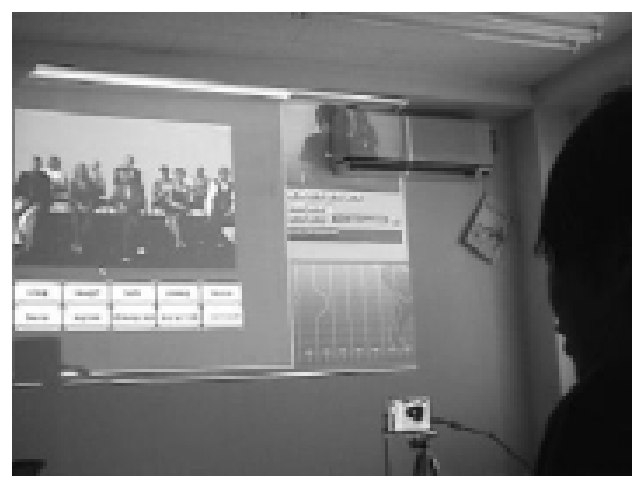

Figure 7. Actor's view

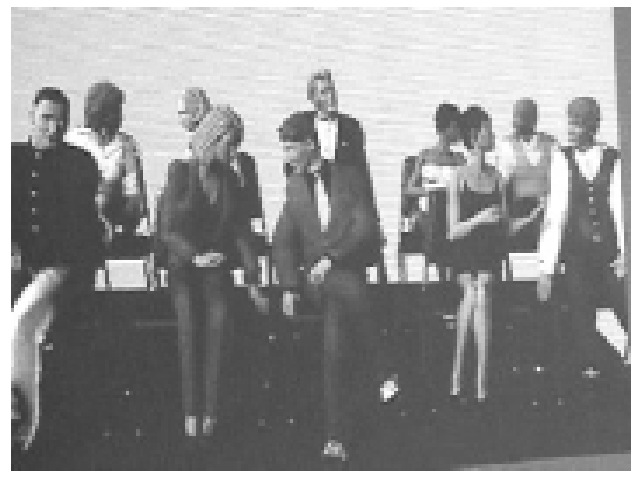

Figure 8. Projected avatars of audiences

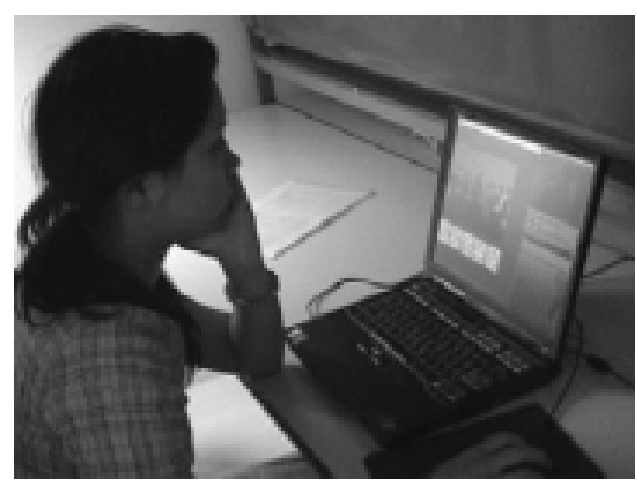

Figure 9. Audience 


\subsubsection{From actors' viewpoint}

The experimental results show that most of the actors could recognize this kind of audience also in the cybertheater. They could really feel as if there were audiences in front of them and could even interact with them.

We also found that for the actors the actions of the avatars are more impressive than the facial expressions of the avatars. This is because when actors are performing, they cannot put too much attention to the audience.

In addition, we got some suggestions from subjects that were not included in our questionnaires. Actors said that the sounds also have a very deep influence on their performances, because sounds are noticed more quickly than actions; sometimes actors just judge the reactions of audiences from their sounds without looking at them. .

\subsubsection{From audiences' viewpoints}

We also got some suggestions from audiences about the model system. First, it is better to make it so that the length of the time during which audiences hold the button can be controlled by the audience. That length is fixed at the moment, and the audience has to wait till one action is finished before they can start pressing the next button. This stops the audience from having the chance to show their real emotions immediately.

Second, many subjects suggested each action should have more variations; e.g. in case of laugh: smile, laugh, laugh heartily, laugh hardly at all and so on. Only one kind of laugh cannot represent their emotions.

The audiences hope the system can be used by multi-users at the same time and that the audiences can chat with each other via this system while they are watching the performance.

The most interesting discovery we found is that the audiences in the Cyber-theater tended to show more intense reactions than in a real theater. The reason might be that the actors do not see the audiences in the cyber-theater directly. Therefore, the audiences tend to feel free to show their real emotions.

\section{SUMMARY AND CONCLUSION}

To explore whether the audience effect, which exists in real space theaters, exists also in Cyber-theaters, the authors conducted some experiments that analyze interactions between actors and audiences in the Cyber-theater. We constructed an experimental system in which remotely located audiences' avatars are displayed to the projector screen that can be seen by actors in real space. In this paper's particular set-up, one audience, whom the two actors in the real space cannot see, can display their emotional actions through his/her avatar by pushing the corresponding button. From the questionnaires we asked actors and audiences to fill out, we obtain the following results.

(1). Most of the actors felt that they were indeed affected by the audiences' avatars. Most of the audiences felt this kind of system is easy to use, but it is quite inconvenient for them to use it while they are watching a performance, because they have to use the mouse to choose an action button.
(2). About the model system, it is better to make the length of time that audiences hold the button to be controlled by audiences. The audiences also claim that each action should have more variations: e.g. currently only laugh, but there should be smile, laugh, laugh slightly and so on.

(3). The audiences in cyber-theater turned to show more intense reactions than in a real theater. The reason might be that the actors do not see the audiences in cyber-theater directly. Therefore, the audiences tend to feel free to show their real emotions.

The audience effect is normally not very explicit except for some of special plays such as comedies and gags: that is, it can be said that the audience effect is caused by psychological sympathy and non-verbal communication between the audiences and the performers. The abovementioned experimental results indicate that the audience effect exists also in the Cyber Theater; in particular, the item (3) supports that the Cyber-theater can emphasize the audience effect even for any kind of plays other than comedies and gags.

In this paper we do not have enough time to put forth the effort on interactions between audiences. Therefore, it will be the next step for us to achieve that in the future.

Moreover, the system should be online so that it can be used by multi-users at the same time. Thus, each avatar can present each individual audience's different actions. Obviously, the "push button" interface is a temporary interface. It should be replaced by a better interface such as a computer vision technology that autonomously recognizes each audience member's actions. The chat functions should be increased because audience might want to discuss with each other.

By doing this, we can make the system more similar to or more effective than the real world.

\section{REFERENCES}

[1] Kang DongWan and Ohya Jun "Study of estimating human body posture from multiple images acquired by hybrid cameras attached and not-attached to the body", 2002 National Convention Record I.E.E.Japan, PP. 48-49 (2002)

[2] M. Slater ET al., IEEE Computer Graphics and Applications, 19(2), PP. 6-9, March/April 1999

[3] Kang DongWan and Ohya Jun "Estimating Postures of a Human Wearing a Multiple Colored Suit Based on Color Information Processing”, 2003 IEEE International Conference on Multimedia and Expo (ICME2003), 4 pages (CD-ROM Proceedings), 2003.

[4] Kang DongWan and Yuichi Onuma, Ohya Jun "Estimating Complicated and Overlapped Human Body Postures by Wearing a Multiple-Colored Suit Using Color Information Processing", 2004 IEEE International Conference on Automatic Face and Gesture Recognition (FGR2004), 6 pages, PP. 687-692 